\title{
Inference of hand movements from local field potentials in monkey motor cortex
}

Carsten Mehring, Jörn Rickert, Eilon Vaadia, Simone Cardoso de Oliveira, Ad Aertsen \& Stefan Rotter Nat. Neurosci. 6, 1253-1254 (2003)

In the version of this brief communication initially published online, the Supplementary Methods file included some incorrect figure callouts. Callouts to Supplementary Fig. 1a-c have now been corrected to read Supplementary Fig. 2a-c, and callouts to Supplementary Fig. 2 have now been corrected to read Supplementary Fig. 1. 